\title{
Mosquito Acetylcholinesterase as a Target for Novel Phenyl-Substituted Carbamates
}

\author{
James M. Mutunga ${ }^{1,+}$, Ming Ma ${ }^{2}$, Qiao-Hong Chen ${ }^{2}{ }^{(0}$, Joshua A. Hartsel ${ }^{2}$, Dawn M. Wong ${ }^{\circledR}$, \\ Sha Ding ${ }^{2}$, Max Totrov ${ }^{3}$, Paul R. Carlier ${ }^{2}(1)$ and Jeffrey R. Bloomquist ${ }^{1, *}$ \\ 1 Emerging Pathogens Institute, Entomology and Nematology Department, University of Florida, \\ Gainesville, FL 32610, USA; James.Mutunga@usamru-k.org \\ 2 Department of Chemistry, Virginia Polytechnic Institute and State University, Blacksburg, VA 24061, USA; \\ gym7981@gmail.com (M.M.); qchen@csufresno.edu (Q.-H.C.); jhartsel@vt.edu (J.A.H.); \\ dawnwong@vt.edu (D.M.W.); sding@vt.edu (S.D.); pcarlier@vt.edu (P.R.C.) \\ 3 Molsoft LLC, 11199 Sorrento Valley Road, S209 San Diego, CA 92121, USA; max@molsoft.com \\ * Correspondence: jbquist@epi.ufl.edu; Tel.: +1-352-273-9417 \\ + Current Address: Department of Entomology, US Army Medical Research Directorate-Africa, Kenya, \\ KEMRI CGHR, P.O. Box 54-40100, Kisumu, Kenya.
}

Received: 26 February 2019; Accepted: 24 April 2019; Published: 28 April 2019

\begin{abstract}
New insecticides are needed for control of disease-vectoring mosquitoes and this research evaluates the activity of new carbamate acetylcholinesterase (AChE) inhibitors. Biochemical and toxicological characterization of carbamates based on the parent structure of terbam, 3-tert-butylphenyl methylcarbamate, was performed. In vitro enzyme inhibition selectivity (Anopheles gambiae versus human) was assessed by the Ellman assay, as well as the lethality to whole insects by the World Health Organization (WHO) paper contact assay. Bromination at the phenyl C6 position increased inhibitory potency to both AChEs, whereas a 6-iodo substituent led to loss of potency, and both halogenations caused a significant reduction of mosquitocidal activity. Similarly, installation of a hexyl substituent at C6 drastically reduced inhibition of $A g A C h E$, but showed a smaller reduction in the inhibition of hAChE. A series of 4-carboxamido analogs of the parent compound gave reduced activity against $A g \mathrm{AChE}$ and generally showed more activity against hAChE than $A g A C h E$. Replacement of the 3-t-buyl group with $\mathrm{CF}_{3}$ resulted in poor anticholinesterase activity, but this compound did have measurable mosquitocidal activity. A series of methyl- and fluoro- analogs of 3-trialkylsilyl compounds were also synthesized, but unfortunately resulted in disappointing activity. Finally, a series of sulfenylated proinsecticides showed poor paper contact toxicity, but one of them had topical activity against adult female Anopheles gambiae. Overall, the analogs prepared here contributed to a better understanding of carbamate structure-activity relationships (SAR), but no new significant leads were generated.
\end{abstract}

Keywords: Anopheles gambiae; anticholinesterase; insecticide; toxicity

\section{Introduction}

The use of commercial carbamate insecticides dates back to the late 1950s, and numerous efforts have been made to improve their toxicity to insects. Despite the wide-scale use of carbamates for crop and urban pest control [1], only propoxur and bendiocarb are authorized by the World Health Organization (WHO) for indoor residual spraying of mosquitoes for malaria control [2]. A major concern for deploying carbamates in or near human dwellings is non-target toxicity, and two primary factors determine the selectivity of a carbamate: selective detoxification in vertebrate systems and selective AChE inhibition in insects [3]. Current advances in proteomics and genomics, the availability of both the Anopheles gambiae and human AChE sequences, and advanced tools of computational 
biology enable fine-scale ligand docking, and virtual ligand library screening aid in the design of new AChE inhibitors.

This study describes our continuing efforts to improve carbamate insecticides for mosquito control, building on our previous work with phenyl-substituted analogs [4-7]. Structural modifications included extending the side-chain branching of alkyl or silyl substituents to explore the effects of spatial and structural complementarity of compounds to the ammonium group of acetylcholine (ACh), since Kolbezen et al. [8] suggested that the structural complementarity of the 3-tert-butyl group of terbam 1a to ACh explained its high potency with bovine AChE. Ring halogenation investigated not only the size of the halogen substituent, but also the preferred placement on the molecule. Carbamate side-chains are conventionally thought to be major detoxication sites [9], and halogenation at these sites typically confers protection against hydroxylation by P450 mono-oxygenases [10]. Inclusion of thioalkyl substituents sought to explore not only the ACh structural complementarity of these analogs, but also the possible bioactivation of sulfur to more toxic sulfoxide and sulfone derivatives, in vivo. These data help to better understand the interactions of carbamates with insect and human AChE and inform the future design of a pharmacophore with superior insect toxicity and selectivity.

\section{Materials and Methods}

\subsection{Preparation of Carbamates}

Carbamates 1a, 3a, 4a, and 5a were prepared as previously described by Hartsel et al. [5] and the synthesis of other experimental compounds is described below.

4-bromo-3-(tert-butyl)phenyl methylcarbamate (1b): 3-t-butylphenol (3 g) was dissolved in 10\% $\mathrm{NaOH}(10 \mathrm{~mL})$, and iodine (5.6 g, 1.1 equivalent) and sodium iodide ( $5.6 \mathrm{~g}, 1.5$ equivalent) were added to afford 5-(t-butyl)-2-iodophenol (4.96 g, 90\% yield). Treatment with bromine in $\mathrm{CH}_{2} \mathrm{Cl}_{2}$ afforded 4-bromo-5-t-butyl-2-iodophenol; refluxing in N-methylmorpholine for $16 \mathrm{~h}$, followed by column chromatography afforded 4-bromo-3-t-butylphenol in 50\% yield over two steps. This compound (50 $\mathrm{mg}$ ) was dissolved in dry tetrahydrofuran (THF) $(2 \mathrm{~mL})$, and potassium t-butoxide $(0.29 \mathrm{~mL}, 1 \mathrm{M}$ in THF) was added followed by methylcarbamoyl chloride (2 equiv). After $1 \mathrm{~h}$, aqueous workup and column chromatography afforded $\mathbf{1 b}$ as a pale semi-solid $(43 \mathrm{mg}, 69 \%) .{ }^{1} \mathrm{H} \mathrm{NMR}\left(400 \mathrm{MHz}, \mathrm{CDCl}_{3}\right) \delta$ $7.53(\mathrm{~d}, J=10.0 \mathrm{~Hz}, 1 \mathrm{H}), 7.19(\mathrm{~s}, 1 \mathrm{H}), 6.85(\mathrm{~d}, J=10.0 \mathrm{~Hz}, 1 \mathrm{H}), 4.99(\mathrm{br} \mathrm{s}, 1 \mathrm{H}), 2.95(\mathrm{~d}, J=3.0 \mathrm{~Hz}, 0.15 \mathrm{H}$, minor carbamate rotamer), $2.88\left(\mathrm{~d}, J=3.0 \mathrm{~Hz}, 2.85 \mathrm{H}\right.$, major carbamate rotamer), $1.50(\mathrm{~s}, 9 \mathrm{H}) ;{ }^{13} \mathrm{C} \mathrm{NMR}$ $\left(101 \mathrm{MHz}, \mathrm{CDCl}_{3}\right) \delta 154.9,150.2,149.2,136.2,121.6,120.7,118.4,36.7,29.5$, and 27.8.

2-bromo-5-(tert-butyl)phenyl methylcarbamate (1d): 3-t-butylphenol (3 g) was dissolved in dichloromethane $(30 \mathrm{~mL})$ and bromine $(1.1 \mathrm{~mL}, 1.1$ equivalent) was added dropwise. After stirring for $1 \mathrm{~h}$, aqueous workup and concentration in vacuo afforded 2-bromo-5-t-butylphenol in quantitative yield. This phenol was treated as above for $\mathbf{1 b}$ to afford $\mathbf{1 d}$ as pale semi-solid (70\% yield). ${ }^{1} \mathrm{H}$ NMR $\left(400 \mathrm{MHz}, \mathrm{CDCl}_{3}\right) \delta 7.44(\mathrm{~d}, J=8.4 \mathrm{~Hz}, 1 \mathrm{H}), 7.16(\mathrm{~d}, J=2.0 \mathrm{~Hz}, 1 \mathrm{H}), 7.08(\mathrm{dd}, J=8.4,2.0 \mathrm{~Hz}, 1 \mathrm{H})$, $5.05(\mathrm{br} \mathrm{s}, 1 \mathrm{H}), 3.00(\mathrm{~d}, J=4.8 \mathrm{~Hz}, 0.15 \mathrm{H}$, minor carbamate rotamer), $2.89(\mathrm{~d}, J=4.8 \mathrm{~Hz}, 2.85 \mathrm{H}$, major carbamate rotamer), $1.26(\mathrm{~s}, 9 \mathrm{H}) ;{ }^{13} \mathrm{C} \mathrm{NMR}\left(101 \mathrm{MHz}, \mathrm{CDCl}_{3}\right) \delta 154.3,152.1,147.9,132.4,124.3,121.3$, 113.0, 34.7, 31.1, and 27.8.

5-(tert-butyl)-2-iodophenyl methylcarbamate (1e): 5-(tert-butyl)-2-iodophenol (described above in synthesis of $1 \mathrm{~b})$ was then dissolved in dry THF $(10 \mathrm{~mL})$, treated with $\mathrm{NaH}(60 \%$ dispersion in mineral oil, 1.6 equivalent), and methylcarbamoyl chloride ( 2.5 equiv). After $1 \mathrm{~h}$ aqueous workup and column chromatography afforded $1 \mathrm{e}$ as a pale semi-solid (134 mg 50\%). ${ }^{1} \mathrm{H} \mathrm{NMR}\left(400 \mathrm{MHz}, \mathrm{CDCl}_{3}\right) \delta 7.68(\mathrm{~d}$, $J=8.3 \mathrm{~Hz}, 1 \mathrm{H}), 7.17(\mathrm{~s}, 1 \mathrm{H}), 6.97(\mathrm{~d}, J=8.3 \mathrm{~Hz}, 1 \mathrm{H}), 5.19(\mathrm{br} \mathrm{s}, 1 \mathrm{H}), 3.01(\mathrm{br} \mathrm{s}, 0.15 \mathrm{H}$, minor carbamate rotamer), 2.89 (br s, $2.85 \mathrm{H}$, major carbamate rotamer), $1.28(\mathrm{~s}, 9 \mathrm{H}) ;{ }^{13} \mathrm{C} \mathrm{NMR}\left(101 \mathrm{MHz}, \mathrm{CDCl}_{3}\right) \delta 154.3$, $153.3,150.8,138.4,124.6,120.6,86.7,34.6,31.1$, and 27.8 .

5-(tert-butyl)-2-hexylphenyl methylcarbamate (1f): The requisite phenol was prepared from 5 -(t-butyl)-2-iodophenol (see above) in a four-step sequence ( $62 \%$ overall yield) by (i) acetylation, (ii) Sonogashira coupling with 1-hexyne, catalytic hydrogenation, and deacetylation. This phenol was then reacted with $\mathrm{NaH}$ and methylcarbamoyl chloride according to the procedure for 1e, to afford 
If as a pale semi-solid (110 mg, 58\%). ${ }^{1} \mathrm{H}$ NMR $\left(400 \mathrm{MHz}, \mathrm{CDCl}_{3}\right) \delta 7.23-7.13(\mathrm{~m}, 2 \mathrm{H}), 7.06(\mathrm{~s}, 1 \mathrm{H})$ $5.01(\mathrm{br} \mathrm{s}, 1 \mathrm{H}), 2.94(\mathrm{~d}, J=4.8 \mathrm{~Hz}, 0.15 \mathrm{H}$, minor carbamate rotamer) $2.88(\mathrm{~d}, J=4.8 \mathrm{~Hz}, 2.85 \mathrm{H}$, major carbamate rotamer), $2.50(\mathrm{t}, J=7.7 \mathrm{~Hz}, 2 \mathrm{H}), 1.61-1.52(\mathrm{~m}, 2 \mathrm{H}), 1.36-1.23(\mathrm{~m}, 15 \mathrm{H}), 0.88(\mathrm{t}, J=6.8 \mathrm{~Hz}$, $1 \mathrm{H}) ;{ }^{13} \mathrm{C}$ NMR $\left(101 \mathrm{MHz}, \mathrm{CDCl}_{3}\right) \delta 155.4,150.0,148.7,131.8,129.4,122.5,119.4,34.4,31.6,31.2,29.9$, 29.6, 29.2, 27.7, 22.5, and 14.1.

4-(allylcarbamoyl)-3-(tert-butyl)phenyl methylcarbamate (1h): 4-bromo-3-t-butylphenol (above in $\mathbf{1 b}$ ) was converted to the triiisopropylsilyl derivative (TIPS-Cl, imidazole, DMF), dissolved in diethyl ether, metalated with $\mathrm{t}-\mathrm{BuLi}$ and trapped with ethylchloroformate, and then hydrolyzed $(\mathrm{KOH}$, aq THF; $\mathrm{HCl}$ aq). Treatment with TBS- $\mathrm{Cl}$ and imidazole in dichloromethane afford 2-(t-butyl)-4-t-butyldimethylsilyloxybenzoic acid in 57\% yield over four steps. Treatment with oxalyl chloride, followed by allyl amine afforded the allyl amide. Deprotection with HF/pyridine, deprotonation with potassium t-butoxide and acylation with methylcarbamoyl chloride afforded $\mathbf{1 h}$ as a pale oil ( $46 \%$ over three steps). ${ }^{1} \mathrm{H}$ NMR $\left(400 \mathrm{MHz}, \mathrm{CDCl}_{3}\right)$ : Note: chiral axis $\mathrm{C} 4-\mathrm{C}(\mathrm{O})$ renders the four $\mathrm{CH}_{2}$ protons diastereotopic $\delta 7.22(\mathrm{~d}, J=9.0 \mathrm{~Hz}, 1 \mathrm{H}), 7.15(\mathrm{~s}, 1 \mathrm{H}), 6.88(\mathrm{~d}, J=9.0 \mathrm{~Hz}, 1 \mathrm{H}) 5.88(\mathrm{ddt}$, $J=15.0,10.0,6.2 \mathrm{~Hz}, 1 \mathrm{H}), 5.81(\mathrm{br} \mathrm{s}, 1 \mathrm{H}), 5.25(\mathrm{dd}, J=15.0,2.0 \mathrm{~Hz}, 1 \mathrm{H}), 5.22(\mathrm{dd}, J=10.0,2.0 \mathrm{~Hz}, 1 \mathrm{H})$, $5.20(\mathrm{br} \mathrm{s}, 1 \mathrm{H}), 4.00(\mathrm{t}, J=6.2 \mathrm{~Hz}, 2 \mathrm{H}), 2.94(\mathrm{~d}, J=4.0 \mathrm{~Hz}, 0.15 \mathrm{H}$, minor carbamate rotamer), $2.82(\mathrm{~d}$, $J=4.0 \mathrm{~Hz}, 2.85 \mathrm{H}$, major carbamate rotamer), $1.45(\mathrm{~s}, 9 \mathrm{H}) ;{ }^{13} \mathrm{C} \mathrm{NMR}\left(101 \mathrm{MHz}, \mathrm{CDCl}_{3}\right) \delta 172.3,155.2$, 151.6, 149.7, 133.7, 133.2, 129.4, 120.6, 118.8, 117.2, 42.5, 36.4, 31.4, and 27.8.

3-(tert-butyl)-4-(methylcarbamoyl)phenyl methylcarbamate (1i): 2-(t-butyl)-4-tbutyldimethylsilyloxybenzoic acid (from $\mathbf{1 b}$ above) was treated with oxalyl chloride, followed by methylamine. Deprotection with $\mathrm{HF} /$ pyridine, deprotonation with potassium t-butoxide and acylation with methylcarbamoyl chloride afforded $1 \mathrm{i}$ as a pale oil (42\% over three steps). ${ }^{1} \mathrm{H}$ NMR $(400 \mathrm{MHz}$, $\left.\mathrm{CDCl}_{3}\right)$ : Note: chiral axis $\mathrm{C} 4-\mathrm{C}(\mathrm{O})$ renders the four $\mathrm{CH}_{2}$ protons diastereotopic $\delta 7.22-7.13(\mathrm{~m}, 2 \mathrm{H}), 6.89(\mathrm{~d}$, $J=9.0 \mathrm{~Hz}, 1 \mathrm{H}), 5.77(\mathrm{br} \mathrm{s}, 1 \mathrm{H}), 5.18(\mathrm{br} \mathrm{s}, 1 \mathrm{H}), 2.93(\mathrm{~d}, J=5.0 \mathrm{~Hz}, 3 \mathrm{H}), 2.85(\mathrm{~d}, J=4.4 \mathrm{~Hz}, 3 \mathrm{H}), 1.37(\mathrm{~s}, 9 \mathrm{H})$; ${ }^{13} \mathrm{C} \mathrm{NMR}\left(101 \mathrm{MHz}, \mathrm{CDCl}_{3}\right) \delta 173.2,155.2,151.5,149.6,133.9,129.3,120.5,118.8,36.3,31.3,27.8$, and 26.9 .

3-(tert-butyl)-4-(isobutylcarbamoyl)phenyl methylcarbamate (1j): 2-(tert-butyl)-4-tbutyldimethylsilyloxybenzoic acid (from $\mathbf{1 b}$ above) was treated with oxalyl chloride, followed by isobutylamine. Deprotection with $\mathrm{HF} /$ pyridine, deprotonation with potassium t-butoxide and acylation with methylcarbamoyl chloride afforded $\mathbf{1 j}$ as a pale oil $\left(28 \%\right.$ over three steps). ${ }^{1} \mathrm{H}$ NMR $\left(400 \mathrm{MHz}, \mathrm{CDCl}_{3}\right)$ : Note: chiral axis $\mathrm{C} 4-\mathrm{C}(\mathrm{O})$ renders the four $\mathrm{CH}_{2}$ protons diastereotopic $\delta 7.22(\mathrm{~d}, J=$ $8.3 \mathrm{~Hz}, 1 \mathrm{H}), 7.18(\mathrm{~s}, 1 \mathrm{H}), 6.95(\mathrm{~d}, J=8.3 \mathrm{~Hz}, 1 \mathrm{H}), 5.80(\mathrm{br} \mathrm{s}, 1 \mathrm{H}), 5.19(\mathrm{br} \mathrm{s}, 1 \mathrm{H}), 3.23(\mathrm{dd}, J=6.2,5.8 \mathrm{~Hz}$, $2 \mathrm{H}), 2.85(\mathrm{~d}, J=3.8 \mathrm{~Hz}, 0.15 \mathrm{H}$, minor carbamate rotamer), $2.79(\mathrm{~d}, J=3.8 \mathrm{~Hz}, 2.85 \mathrm{H}$, major carbamate rotamer), 1.90 (t sep, $J=6.8,6.2 \mathrm{~Hz}, 1 \mathrm{H}), 1.44(\mathrm{~s}, 9 \mathrm{H}), 0.92(\mathrm{~d}, J=6.8 \mathrm{~Hz}, 6 \mathrm{H}) ;{ }^{13} \mathrm{C}$ NMR $(101 \mathrm{MHz}$, $\left.\mathrm{CDCl}_{3}\right) \delta 172.6,155.2,151.5,149.6,134.1,129.4,120.5,118.8,47.6,36.3,31.3,28.3,27.8$, and 20.3.

3-(tert-butyl)-4-(diethylcarbamoyl)phenyl methylcarbamate

(1k): 2-(t-butyl)-4-t-butyldimethylsilyloxybenzoic acid (from $\mathbf{1 b}$ above) was treated with oxalyl chloride, followed by diethylamine. Deprotection with HF/pyridine, deprotonation with potassium t-butoxide and acylation with methylcarbamoyl chloride afforded $1 \mathrm{k}$ as a pale oil ( $29 \%$ over three steps). ${ }^{1} \mathrm{H}$ NMR (400 MHz, $\mathrm{CDCl}_{3}$ ): Note: chiral axis $\mathrm{C} 4-\mathrm{C}(\mathrm{O})$ renders the four $\mathrm{CH}_{2}$ protons diastereotopic $\delta$ $7.20(\mathrm{~s}, 1 \mathrm{H}), 6.98(\mathrm{~d}, J=8.0 \mathrm{~Hz}, 1 \mathrm{H}), 6.83(\mathrm{~d}, J=8.0 \mathrm{~Hz}, 1 \mathrm{H}), 5.05(\mathrm{br} \mathrm{s}, 1 \mathrm{H}), 3.72(\mathrm{dq}, J=15.2,6.2 \mathrm{~Hz}$, $1 \mathrm{H}), 3.28(\mathrm{dq}, J=15.6,6.2 \mathrm{~Hz}, 1 \mathrm{H}), 3.20(\mathrm{dq}, J=15.2,6.2 \mathrm{~Hz}, 1 \mathrm{H}), 3.01(\mathrm{dq}, J=15.6,6.2 \mathrm{~Hz}, 1 \mathrm{H}), 2.95(\mathrm{~d}$, $J=4.0 \mathrm{~Hz}, 0.15 \mathrm{H}$, minor carbamate rotamer), $2.78(\mathrm{~d}, J=4.0 \mathrm{~Hz}, 2.85 \mathrm{H}$, major carbamate rotamer), 1.29 $(\mathrm{s}, 9 \mathrm{H}), 1.23(\mathrm{dd}, J=6.2,6.2 \mathrm{~Hz}, 3 \mathrm{H}), 1.02(\mathrm{dd}, J=6.2,6.2 \mathrm{~Hz}, 3 \mathrm{H}) ;{ }^{13} \mathrm{C} \mathrm{NMR}\left(101 \mathrm{MHz}, \mathrm{CDCl}_{3}\right) \delta 172.3$, 154.6, 150.8, 147.0, 133.5, 127.7, 120.9, 118.1, 44.2, 38.6, 36.1, 32.0, 27.5, 13.8, and 12.7.

3-(tert-butyl)-4-(ethyl(methyl)carbamoyl)phenyl methylcarbamate (11): 2-(t-butyl)-4-tbutyldimethylsilyloxybenzoic acid (from $\mathbf{1 b}$ above) was treated with oxalyl chloride, followed by ethylmethylamine. Deprotection with HF/pyridine, deprotonation with potassium t-butoxide and acylation with methylcarbamoyl chloride afforded 11 as a pale oil (59\% over three steps). ${ }^{1} \mathrm{H}$ NMR $\left(400 \mathrm{MHz}, \mathrm{CDCl}_{3}\right)$ : Note: chiral axis $\mathrm{C} 4-\mathrm{C}(\mathrm{O})$ renders the four $\mathrm{CH}_{2}$ protons diastereotopic $\delta 7.13(\mathrm{~d}$, $J=1.8 \mathrm{~Hz}, 1 \mathrm{H}), 7.02(\mathrm{dd}, J=8.2,1.8 \mathrm{~Hz}, 1 \mathrm{H}), 6.87(\mathrm{~d}, J=8.2 \mathrm{~Hz}, 1 \mathrm{H}), 5.32(\mathrm{br} \mathrm{s}, 1 \mathrm{H}), 3.69(\mathrm{dt}, J=15.2$, 
$6.8 \mathrm{~Hz}, 0.5 \mathrm{H}), 3.48(\mathrm{dt}, J=15.2,6.8 \mathrm{~Hz}, 0.5 \mathrm{H}), 3.25(\mathrm{dt}, J=15.2,6.8 \mathrm{~Hz}, 0.5 \mathrm{H}), 3.05(\mathrm{~s}, 1.5 \mathrm{H}), 2.98(\mathrm{dt}, J$ $=15.2,6.8 \mathrm{~Hz}, 0.5 \mathrm{H}), 2.97(\mathrm{~d}, J=3.8 \mathrm{~Hz}, 0.15 \mathrm{H}$, minor carbamate rotamer), $2.82(\mathrm{~d}, J=3.8 \mathrm{~Hz}, 2.85$ $\mathrm{H}$, major carbamate rotamer), $2.75(\mathrm{~s}, 1.5 \mathrm{H}), 1.32(\mathrm{~s}, 9 \mathrm{H}), 1.20(\mathrm{dd}, J=6.8,6.8 \mathrm{~Hz}, 1.5 \mathrm{H}), 0.97(\mathrm{dd}$, $J=6.8,6.8 \mathrm{~Hz}, 1.5 \mathrm{H}) ;{ }^{13} \mathrm{C} \mathrm{NMR}\left(101 \mathrm{MHz}, \mathrm{CDCl}_{3}\right) \delta 176.0,175.0,155.3,151.3,151.2,148.1,148.0,132.6$, $131.9,128.5,127.8,120.9,119.1,118.9,46.0,41.8,36.9,36.4,31.7,31.2,27.7,13.0$, and 11.4 (two equally populated rotamers (ethymethylamide); 24 of a possible 28 resonances seen).

5-(tert-butyl)-2-(methylthio)phenyl methylcarbamate (1m): 3-t-butylphenol was treated with chlorosulfonic acid (10 equivalent) in dichloromethane for $4 \mathrm{~h}$, and then poured into ice. Extractive workup, reduction with stannous chloride in acetic acid (18 h), and column chromatography afforded 6,6'-disulfanediylbis(3-(t-butyl)phenol). Reduction with sodium borohydride in THF gave 5 -(t-butyl)-2-mercaptophenol in $64 \%$ yield over three steps. Treatment with methyl iodide (1.1 equiv) and sodium bicarbonate in DMF, followed by deprotonation with potassium t-butoxide in THF and acylation with methylcarbamoyl chloride gave $1 \mathrm{~m}$ as a pale semi-solid in $37 \%$ yield over two steps. ${ }^{1} \mathrm{H}$ NMR $\left(400 \mathrm{MHz}, \mathrm{CDCl}_{3}\right) \delta 7.23(\mathrm{dd}, J=6.6,1.6 \mathrm{~Hz}, 1 \mathrm{H}), 7.19(\mathrm{~d}, J=6.6 \mathrm{~Hz}, 1 \mathrm{H}), 7.12(\mathrm{~d}, J=1.6 \mathrm{~Hz}$, $1 \mathrm{H}), 5.04(\mathrm{br} \mathrm{s}, 1 \mathrm{H}), 3.07(\mathrm{~d}, J=4.0 \mathrm{~Hz}, 0.15 \mathrm{H}$, minor carbamate rotamer), $2.91(\mathrm{~d}, J=4.0 \mathrm{~Hz}, 2.85 \mathrm{H}$, major carbamate rotamer), $2.42(\mathrm{~s}, 3 \mathrm{H}), 1.30(\mathrm{~s}, 9 \mathrm{H}) ;{ }^{13} \mathrm{C} \mathrm{NMR}\left(101 \mathrm{MHz}, \mathrm{CDCl}_{3}\right) \delta 154.9,150.1,148.3$, $128.1,127.3,123.5,120.2,34.6,31.4,28.0,15.7$.

5-(tert-butyl)-2-(isobutylthio)phenyl methylcarbamate (1n): 5-(t-butyl)-2-mercaptophenol (from $1 \mathrm{~m}$ above) was treated with isobutyl iodide (2.9 equivalents) and sodium bicarbonate in DMF at $55^{\circ} \mathrm{C}$ for 6 $\mathrm{h}$, followed by deprotonation with potassium t-butoxide in THF and acylation with methylcarbamoyl chloride to gave $1 \mathrm{n}$ as a pale semi-solid in $63 \%$ yield over two steps. ${ }^{1} \mathrm{H} \mathrm{NMR}\left(400 \mathrm{MHz}, \mathrm{CDCl}_{3}\right) \delta 7.27(\mathrm{~d}$, $J=6.6 \mathrm{~Hz}, 1 \mathrm{H}), 7.18(\mathrm{~d}, J=6.6,1.6 \mathrm{~Hz}, 1 \mathrm{H}), 7.12(\mathrm{~d}, J=1.6 \mathrm{~Hz}, 1 \mathrm{H}), 5.04(\mathrm{br} \mathrm{s}, 1 \mathrm{H}), 3.02,(\mathrm{~d}, J=3.9 \mathrm{~Hz}$, $0.15 \mathrm{H}$, minor carbamate rotamer), $2.92(\mathrm{~d}, J=3.9 \mathrm{~Hz}, 2.85 \mathrm{H}$, major carbamate rotamer), $2.73(\mathrm{~d}, J=5.5 \mathrm{~Hz}$, 2H), 1.81 (nonet (9-let), $J=5.5 \mathrm{~Hz}, 1 \mathrm{H}), 1.29(\mathrm{~s}, 9 \mathrm{H}), 1.02(\mathrm{~J}=5.3 \mathrm{~Hz}, 6 \mathrm{H}) ;{ }^{13} \mathrm{C} \mathrm{NMR}\left(101 \mathrm{MHz}, \mathrm{CDCl}_{3}\right) \delta$ 155.0, 150.9, 149.5, 130.1, 127.0, 123.4, 120.3, 42.5, 34.7, 31.3, 28.4, 28.0, 22.2.

5-(tert-butyl)-2-(isopropylthio)phenyl methylcarbamate (10): 5-(t-butyl)-2-mercaptophenol (from $1 \mathrm{~m}$ above) was treated with isopropyl iodide (3.0 equiv) and sodium bicarbonate in DMF at $55{ }^{\circ} \mathrm{C}$ for $6 \mathrm{~h}$, followed by deprotonation with potassium t-butoxide in THF and acylation with methylcarbamoyl chloride to give 10 as a pale semi-solid in $60 \%$ yield over two steps. ${ }^{1} \mathrm{H} \mathrm{NMR}\left(400 \mathrm{MHz}, \mathrm{CDCl}_{3}\right) \delta$ $7.36(\mathrm{~d}, J=6.6 \mathrm{~Hz}, 1 \mathrm{H}), 7.19(\mathrm{dd}, J=6.6,1.6 \mathrm{~Hz}, 1 \mathrm{H}), 7.14(\mathrm{~d}, J=1.6 \mathrm{~Hz}, 1 \mathrm{H}), 5.03(\mathrm{br} \mathrm{s}, 1 \mathrm{H}), 3.33(\mathrm{sept}$, $J=5.3 \mathrm{~Hz}, 1 \mathrm{H}), 3.02(\mathrm{~d}, J=3.9 \mathrm{~Hz}, 0.15 \mathrm{H}$, minor carbamate rotamer), $2.92(\mathrm{~d}, J=3.9 \mathrm{~Hz}, 2.85 \mathrm{H}$, major carbamate rotamer), $1.30(\mathrm{~s}, 9 \mathrm{H}), 1.27(\mathrm{~d}, J=5.3 \mathrm{~Hz}, 6 \mathrm{H}) ;{ }^{13} \mathrm{C} \mathrm{NMR}\left(101 \mathrm{MHz}, \mathrm{CDCl}_{3}\right) \delta$ 155.1, 152.1, $150.7,133.2,125.3,134.3,120.4,37.9,34.8,31.3,28.0$, and 23.4.

3-(trifluoromethyl)phenyl methylcarbamate (2a): 3-trifluoromethylphenol (1.09 g) was deprotonated with potassium t-butoxide in THF and treated with methylcarbamoyl chloride to give $2 \mathrm{a}$ as a pale semi-solid (1.16 g, 81\% yield). ${ }^{1} \mathrm{H}$ NMR $\left(400 \mathrm{MHz}, \mathrm{CDCl}_{3}\right)$ 8 7.47-7.44 (m, $\left.2 \mathrm{H}\right), 7.40$ (br $\mathrm{s}, 1 \mathrm{H}), 7.33-7.31(\mathrm{~m}, 1 \mathrm{H}), 5.17(\mathrm{br} \mathrm{s}, 1 \mathrm{H}), 2.95(\mathrm{~d}, J=4.0 \mathrm{~Hz}, 0.15 \mathrm{H}$, minor carbamate rotamer), $2.88(\mathrm{~d}$, $J=4.0 \mathrm{~Hz}, 2.85 \mathrm{H}$, major carbamate rotamer); ${ }^{13} \mathrm{C} \mathrm{NMR}\left(101 \mathrm{MHz}, \mathrm{CDCl}_{3}\right) \delta 154.8,151.3,132.3,129.9$, $125.3,123.3\left(\mathrm{q},{ }^{1} \mathrm{~J}_{\mathrm{CF}}=238 \mathrm{~Hz}\right), 122.1,118.9$, and 27.8 .

4-methyl-3-(trimethylsilyl)phenyl methylcarbamate (3c): 3-bromo-4-methylphenol (0.70 g) was dissolved in dry THF $(5 \mathrm{~mL})$, cooled to $-78^{\circ} \mathrm{C}$, treated with BuLi $(3.4 \mathrm{~mL}, 2.5 \mathrm{M}$ in hexanes, 2.2 equivalent) and trimethylsilyl chloride $(1.2 \mathrm{~mL}, 1.1 \mathrm{~g}$, 2.6 equivalent). After stirring for $30 \mathrm{~min}$ the reaction was allowed to warm to $25^{\circ} \mathrm{C}$ and the reaction was quenched with aqueous $\mathrm{HCl}$. Extractive workup and column chromatograph afforded 4-methyl-3-trimethylsilylphenol as a colorless oil $(630 \mathrm{mg}$, 93\%). Applying the procedure above, the phenol $(630 \mathrm{mg})$ was converted to the methyl carbamate, affording $3 \mathrm{c}$ as a yellow oil (591 mg, $71 \%$ yield). ${ }^{1} \mathrm{H} \mathrm{NMR}\left(400 \mathrm{MHz}, \mathrm{CDCl}_{3}\right) \delta 7.12(\mathrm{~s}, 1 \mathrm{H}), 7.11(\mathrm{~d}$, $J=9.6 \mathrm{~Hz}, 1 \mathrm{H}), 6.98(\mathrm{~d}, J=9.6 \mathrm{~Hz}, 1 \mathrm{H}), 4.97(\mathrm{br} \mathrm{s}, 1 \mathrm{H}), 2.93(\mathrm{~d}, J=4.0 \mathrm{~Hz}, 0.15 \mathrm{H}$, minor carbamate rotamer), $2.85\left(\mathrm{~d}, J=4.0 \mathrm{~Hz}, 2.85 \mathrm{H}\right.$, major carbamate rotamer), $2.40(\mathrm{~s}, 3 \mathrm{H}), 0.30(\mathrm{~s}, 9 \mathrm{H}) ;{ }^{13} \mathrm{C} \mathrm{NMR}$ $\left(101 \mathrm{MHz}, \mathrm{CDCl}_{3}\right) \delta 155.8,148.7,140.6,140.1,130.8,127.1,122.4,27.9,22.5$, and -0.13 . 
3-fluoro-5-(trimethylsilyl)phenyl methylcarbamate (3g): 3-bromo-5-fluorophenol (0.19 g) was dissolved in dry THF $(9 \mathrm{~mL})$, cooled to $-78{ }^{\circ} \mathrm{C}$, treated with BuLi $(1.4 \mathrm{~mL}, 2.5 \mathrm{M}$ in hexanes, 3.3 equivalent) and trimethylsilyl chloride $(0.45 \mathrm{~mL}, 0.39 \mathrm{~g}$, 3.6 equivalent). After stirring for $30 \mathrm{~min}$ the reaction was allowed to warm to $25^{\circ} \mathrm{C}$ and the reaction was quenched with aqueous $\mathrm{HCl}$. Extractive workup and column chromatograph afforded 5-fluoro-3-trimethylsilylphenol as a colorless oil (66 mg, $66 \%)$. Applying the procedure above, the phenol $(66 \mathrm{mg})$ was converted to the methyl carbamate, affording $3 \mathrm{~g}$ as a yellow oil $\left(78 \mathrm{mg}, 92 \%\right.$ yield). ${ }^{1} \mathrm{H}$ NMR $\left(400 \mathrm{MHz}, \mathrm{CDCl}_{3}\right) \delta 7.08-6.99(\mathrm{~m}, 2 \mathrm{H}), 6.87$ $(\mathrm{ddd}, J=9.6,2.3,2.1 \mathrm{~Hz}, 1 \mathrm{H}), 5.12(\mathrm{br} \mathrm{s}, 1 \mathrm{H}), 2.92(\mathrm{~d}, J=4.7 \mathrm{~Hz}, 0.15 \mathrm{H}$, minor carbamate rotamer), 2.87 $\left(\mathrm{d}, J=4.7 \mathrm{~Hz}, 2.85 \mathrm{H}\right.$, major carbamate rotamer), $0.25(\mathrm{~s}, 9 \mathrm{H}) ;{ }^{13} \mathrm{C} \mathrm{NMR}\left(101 \mathrm{MHz}, \mathrm{CDCl}_{3}\right) \delta 162.5(\mathrm{~d}$, $\left.{ }^{1} \mathrm{~J}_{\mathrm{CF}}=248.4 \mathrm{~Hz}\right) 154.9,151.5,144.2\left(\mathrm{~d},{ }^{3} \mathrm{~J}_{\mathrm{CF}}=4.7 \mathrm{~Hz}\right), 121.6\left(\mathrm{~d},{ }^{3} \mathrm{~J}_{\mathrm{CF}}=2.9 \mathrm{~Hz}\right), 116.5\left(\mathrm{~d},{ }^{2} \mathrm{~J}_{\mathrm{CF}}=18.2 \mathrm{~Hz}\right)$, $110.0\left(\mathrm{~d},{ }^{2} \mathrm{~J}_{\mathrm{CF}}=24.1 \mathrm{~Hz}\right), 27.7$, and -1.4 .

3-(ethyldimethylsilyl)-4-methylphenyl methylcarbamate (4c): 3-bromo-4-methylphenol (0.70 g) was dissolved in dry THF $(5 \mathrm{~mL})$, cooled to $-78^{\circ} \mathrm{C}$, treated with BuLi $(3.4 \mathrm{~mL}, 2.5 \mathrm{M}$ in hexanes, 2.2 equivalent) and ethyldimethylsilyl chloride $(1.36 \mathrm{~mL}, 1.19 \mathrm{~g}, 2.6$ equivalent). After stirring for 30 $\mathrm{min}$, the reaction was allowed to warm to $25^{\circ} \mathrm{C}$ and the reaction was quenched with aqueous $\mathrm{HCl}$. Extractive workup and column chromatograph afforded 4-methyl-3-trimethylsilylphenol as a colorless oil ( $665 \mathrm{mg}, 91 \%)$. Applying the procedure above, the phenol $(419 \mathrm{mg})$ was converted to the methyl carbamate, affording $4 \mathrm{c}$ as a yellow oil $\left(419 \mathrm{mg}, 77 \%\right.$ yield). ${ }^{1} \mathrm{H}$ NMR $\left(400 \mathrm{MHz}, \mathrm{CDCl}_{3}\right) \delta 7.13(\mathrm{~s}, 1 \mathrm{H})$, $7.11(\mathrm{~d}, J=9.0 \mathrm{~Hz}, 1 \mathrm{H}), 6.99(\mathrm{~d}, J=9.0 \mathrm{~Hz}, 1 \mathrm{H}), 5.05(\mathrm{br} \mathrm{s}), 2.90(\mathrm{~d}, J=4.9 \mathrm{~Hz}, 0.15 \mathrm{H}$, minor carbamate rotamer), $2.83(\mathrm{~d}, J=4.9 \mathrm{~Hz}, 2.85 \mathrm{H}$, major carbamate rotamer), $2.40(\mathrm{~s}, 3 \mathrm{H}), 0.93(\mathrm{t}, J=8.0 \mathrm{~Hz}, 3 \mathrm{H}), 0.79$ $(\mathrm{q}, J=8.0 \mathrm{~Hz}, 2 \mathrm{H}), 0.28(\mathrm{~s}, 6 \mathrm{H}) ;{ }^{13} \mathrm{C}$ NMR $\left(101 \mathrm{MHz}, \mathrm{CDCl}_{3}\right) \delta 155.9,148.7,140.7,139.2,130.8,127.5$, $122.3,27.9,22.5,7.75,7.74$, and -2.35 .

3-(ethyldimethylsilyl)-5-fluorophenyl methylcarbamate (4g): 3-bromo-5-fluorophenol (0.27 g) was dissolved in dry THF $(9 \mathrm{~mL})$, cooled to -78 Ç, treated with BuLi $(2.1 \mathrm{~mL}, 2.5 \mathrm{M}$ in hexanes, 3.5 equivalent) and ethyldimethylsilyl chloride $(0.80 \mathrm{~mL}, 0.70 \mathrm{~g}, 4.0$ equivalent). After stirring for 30 min the reaction was allowed to warm to $25^{\circ} \mathrm{C}$ and the reaction was quenched with aqueous $\mathrm{HCl}$. Extractive workup and column chromatograph afforded 3-ethyldimethylsilyl-5-fluorophenol as a colorless oil (178 mg, 62\%). Applying the procedure above, the phenol (58 mg) was converted to the methylcarbamate, affording $4 \mathrm{~g}$ as a yellow oil ( $55 \mathrm{mg}, 74 \%$ yield). ${ }^{1} \mathrm{H} \mathrm{NMR}\left(400 \mathrm{MHz}, \mathrm{CDCl}_{3}\right) \delta$ 7.08-6.98 (m, 2H), $6.87(\mathrm{ddd}, J=9.6,2.2,2.2 \mathrm{~Hz}, 1 \mathrm{H}), 5.09(\mathrm{br} \mathrm{s}, 1 \mathrm{H}), 2.93(\mathrm{~d}, J=4.0 \mathrm{~Hz}, 0.15 \mathrm{H}$, minor carbamate rotamer), $2.87(\mathrm{~d}, J=4.0 \mathrm{~Hz}, 2.85 \mathrm{~Hz}$, major carbamate rotamer), $0.94(\mathrm{t}, J=7.8 \mathrm{~Hz}, 3 \mathrm{H}), 0.70$ $(\mathrm{q}, J=7.8 \mathrm{~Hz}, 2 \mathrm{H}), 0.23(\mathrm{~s}, 9 \mathrm{H}) ;{ }^{13} \mathrm{C} \mathrm{NMR}\left(101 \mathrm{MHz}, \mathrm{CDCl}_{3}\right) \delta 162.5\left(\mathrm{~d},{ }^{1} \mathrm{~J}_{\mathrm{CF}}=248.4 \mathrm{~Hz}\right), 154.8,151.5(\mathrm{~d}$, $\left.{ }^{2} \mathrm{~J}_{\mathrm{CF}}=9.7 \mathrm{~Hz}\right), 143.2\left(\mathrm{~d},{ }^{3} \mathrm{~J}_{\mathrm{CF}}=4.6 \mathrm{~Hz}\right), 121.8\left(\mathrm{~d},{ }^{3} \mathrm{~J}_{\mathrm{CF}}=2.9 \mathrm{~Hz}\right), 116.6,109.9\left(\mathrm{~d},{ }^{2} \mathrm{~J}_{\mathrm{CF}}=24.2\right), 27.7,7.23$, 7.13 , and -3.73 .

2-(propylthio)phenyl methylcarbamate (6a) 2-mercaptophenol (300 mg, $2.5 \mathrm{mmol}$ ) was dissolved in dry DMF $(5 \mathrm{~mL})$ and $\mathrm{NaHCO}_{3}(630 \mathrm{mg}$, 3 equivalent) and propyl bromide ( $0.45 \mathrm{~g}, 5.0$ equivalent) were added. After heating to $55{ }^{\circ} \mathrm{C}$ for $16 \mathrm{~h}$, aqueous workup, and column chromatography, 2-propylthiophenol was isolated as a pale oil (391 mg, 98\%). 2-propylthiophenol (354 mg, $2.11 \mathrm{mmol}$ ) was dissolved in dry THF (20 mL), treated with $\mathrm{NaH}(60 \%$ in mineral oil, $110 \mathrm{mg}, 2.75$ equivalent), and methylcarbamoyl chloride ( $394 \mathrm{mg}, 4.2$ equivalent) was added. Aqueous workup and column chromatography afforded $6 \mathrm{a}$ as a pale oil $(360 \mathrm{mg}, 76 \%) .{ }^{1} \mathrm{H} \mathrm{NMR}\left(400 \mathrm{MHz}, \mathrm{CDCl}_{3}\right) \delta 7.33(\mathrm{dd}, J=5.6$, $1.8 \mathrm{~Hz}, 1 \mathrm{H}), 7.21-7.15(\mathrm{~m}, 2 \mathrm{H}), 7.11(\mathrm{dd}, J=5.3,1.9 \mathrm{~Hz}, 1 \mathrm{H}), 5.08(\mathrm{br} \mathrm{s}, 1 \mathrm{H}), 3.02(\mathrm{~d}, J=4.0 \mathrm{~Hz}, 0.15 \mathrm{H}$, minor carbamate rotamer), $2.91(\mathrm{~d}, J=4.0 \mathrm{~Hz}, 2.85 \mathrm{~Hz}$, major carbamate rotamer $), 2.85(\mathrm{t}, J=5.8 \mathrm{~Hz}$, 2H), 1.66 (hextet, $J=5.9 \mathrm{~Hz}, 2 \mathrm{H}), 1.02(\mathrm{t}, J=5.9 \mathrm{~Hz}, 3 \mathrm{H}) ;{ }^{13} \mathrm{C} \mathrm{NMR}\left(101 \mathrm{MHz}, \mathrm{CDCl}_{3}\right) \delta 154.8,149.3$, $130.5,129.5,126.7,126.2,123.1,34.9,28.0,22.5$, and 13.6.

3-(tert-butyl)phenyl methyl(phenylthio)carbamate (7a): Compound 1a (150 mg) was treated with triethylamine (4 equiv) and benzenesulfenyl chloride (1.5 equvalent) in carbon tetrachloride $(4 \mathrm{~mL})$ at $45^{\circ} \mathrm{C}$ for $18 \mathrm{~h}$. Following aqueous workup the residue was chromatographed in 30:1 hexanes:ethyl acetate to afford $7 \mathrm{a}$ as a yellow oil $\left(186 \mathrm{mg}, 82 \%\right.$ yield). ${ }^{1} \mathrm{H}$ NMR $\left(400 \mathrm{MHz}, \mathrm{CDCl}_{3}\right) \delta 7.40-7.36(\mathrm{~m}, 2 \mathrm{H})$, $7.34(\mathrm{~d}, J=9.5 \mathrm{~Hz}, 2 \mathrm{H}), 7.30-7.22(\mathrm{~m}, 3 \mathrm{H}), 7.08(\mathrm{~s}, 1 \mathrm{H}), 6.92(\mathrm{~d}, J=8.2 \mathrm{~Hz}, 1 \mathrm{H}), 3.43(\mathrm{~s}, 3 \mathrm{H}), 1.30(\mathrm{~s}, 9 \mathrm{H})$; 
${ }^{13} \mathrm{C}$ NMR $\left(101 \mathrm{MHz}, \mathrm{CDCl}_{3}\right) \delta 156.8,153.0,151.3,137.5,129.3,128.8,127.3,126.3,125.3,122.8,118.5$, $42.0,34.8$, and 32.2 .

3-(tert-butyl)phenyl methyl(p-tolylthio)carbamate (7b): Following the procedure for $\mathbf{7 a}, \mathbf{1} \mathbf{a}$, and p-toluylsulfenyl chloride were reacted to afford $7 \mathrm{~b}$ as a yellow oil $(213 \mathrm{mg}, 89 \%) .{ }^{1} \mathrm{H} \mathrm{NMR}(400 \mathrm{MHz}$, $\left.\mathrm{CDCl}_{3}\right) \delta 7.36-7.22(\mathrm{~m}, 6 \mathrm{H}), 7.11(\mathrm{~s}, 1 \mathrm{H}), 6.96(\mathrm{br} \mathrm{s}, 1 \mathrm{H}), 3.42(\mathrm{~s}, 3 \mathrm{H}), 2.38(\mathrm{~s}, 3 \mathrm{H}), 1.32(\mathrm{~s}, 9 \mathrm{H}) ;{ }^{13} \mathrm{C} \mathrm{NMR}$ $\left(101 \mathrm{MHz}, \mathrm{CDCl}_{3}\right) \delta 156.8,154.9,151.4,138.2,134.1,130.0,128.8,127.2,122.7,118.6,41.9,34.8,31.3$, and 21.2.

3-(tert-butyl)phenyl ((4-(tert-butyl)phenyl)thio)(methyl)carbamate (7c): following the procedure for $7 \mathbf{a}, 1 \mathbf{a}$, and 4-t-butylphenylsulfenyl chloride were reacted to afford $7 \mathrm{c}$ as a yellow oil $(152 \mathrm{mg}(73 \%)$. ${ }^{1} \mathrm{H}$ NMR $\left(400 \mathrm{MHz}, \mathrm{CDCl}_{3}\right) \delta 7.43(\mathrm{~d}, J=9.7 \mathrm{~Hz}, 2 \mathrm{H}), 7.37(\mathrm{~d}, J=9.7 \mathrm{~Hz}, 2 \mathrm{H}), 7.30(\mathrm{t}, J=8.0 \mathrm{~Hz}, 1 \mathrm{H})$, $7.25(\mathrm{~d}, 8.0 \mathrm{~Hz}, 1 \mathrm{H}), 7.10(\mathrm{~s}, 1 \mathrm{H}), 6.95(\mathrm{~d}, J=8.0 \mathrm{~Hz}, 1 \mathrm{H}), 3.42(\mathrm{~s}, 3 \mathrm{H}), 1.34(\mathrm{~s}, 9 \mathrm{H}), 1.31(\mathrm{~s}, 9 \mathrm{H}) ;{ }^{13} \mathrm{C} \mathrm{NMR}$ $\left(101 \mathrm{MHz}, \mathrm{CDCl}_{3}\right) \delta 156.8,152.9,151.4,151.2,134.3,128.8,126.9,126.3,122.7,118.6,41.9,34.8,34.7,31.6$, $31.3,31.2,22.7$, and 14.1 .

3-(trimethylsilyl)phenyl methyl(phenylthio)carbamate (7d): Following the procedure for $\mathbf{7 a}, \mathbf{3 a}$ and benzenesulfenyl chloride were reacted to afford $7 \mathrm{~d}$ as a yellow oil $(68 \mathrm{mg}, 58 \%) .{ }^{1} \mathrm{H}$ NMR $(400 \mathrm{MHz}$, $\left.\mathrm{CDCl}_{3}\right)$ 8 7.44-7.30 (m, 6H), 7.28-7.23 (m, 1H), $7.19(\mathrm{~s}, 1 \mathrm{H}), 7.09(\mathrm{~s}, 1 \mathrm{H}), 3.47(\mathrm{~s}, 3 \mathrm{H}), 0.27(\mathrm{~s}, 9 \mathrm{H}) ;{ }^{13} \mathrm{C} \mathrm{NMR}$ $\left(101 \mathrm{MHz}, \mathrm{CDCl}_{3}\right) \delta 156.9,151.2,142.7,133.7,130.7,129.4,129.0,127.5,125.9,122.0,42.1$, and -1.11 .

4-methyl-3-(trimethylsilyl)phenyl methyl(phenylthio)carbamate (7e): Following the procedure for $7 \mathrm{a}, 4 \mathrm{c}$ and benzenesulfenyl chloride were reacted to afford $7 \mathbf{e}$ as a yellow oil (166 mg, 58\%). ${ }^{1} \mathrm{H} \mathrm{NMR}$ $\left(400 \mathrm{MHz}, \mathrm{CDCl}_{3}\right) \delta 7.40(\mathrm{~d}, J=8.7 \mathrm{~Hz}, 2 \mathrm{H}), 7.39-7.35(\mathrm{~m}, 2 \mathrm{H}), 7.29(\mathrm{t}, J=8.7, \mathrm{~Hz}, 1 \mathrm{H}), 7.19-7.10(\mathrm{~m}$, $2 \mathrm{H}), 7.00(\mathrm{~d}, J=8.0 \mathrm{~Hz}, 1 \mathrm{H}), 3.44(\mathrm{~s}, 3 \mathrm{H}), 2.44(\mathrm{~s}, 3 \mathrm{H}), 0.27(\mathrm{~s}, 9 \mathrm{H}) ;{ }^{13} \mathrm{C} \mathrm{NMR}\left(101 \mathrm{MHz}, \mathrm{CDCl}_{3}\right) \delta 157.0$, $149.0,140.9,140.1,130.7,129.3,129.0,127.3,126.7,125.6,122.0,42.0,22.3$, and -0.4 .

\subsection{Insects and Reagents}

Anopheles gambiae (insecticide-susceptible G3 strain), were taken from colonies cultured in the Department of Entomology at Virginia Polytechnic Institute and State University, or the University of Florida, Emerging Pathogens Institute. Acetylthiocholine (ATChI), recombinant human enzyme (hAChE), 5,5'-dithio-bis-(2-nitrobenzoic acid) (DTNB), and all buffer components were purchased from Sigma-Aldrich (MO, USA). Whole mosquito homogenate was prepared essentially as described by Anderson et al. [11], and was used as the source of $A g A C h E$.

\subsection{AChE Inhibition Assays}

AChE enzyme inhibition assays were performed using the Ellman [12] method adapted for a 96-well microplate assay [11], with a few modifications. Briefly, inhibitor stocks of $0.01 \mathrm{M}$ were freshly prepared in dimethylsulfoxide (DMSO) followed by serial dilutions in DMSO. A 100-fold dilution into sodium phosphate buffer ( $\mathrm{pH} 7.8$ ) was made for each of the DMSO dilutions. The final DMSO concentration was maintained at $0.1 \% \mathrm{v} / \mathrm{v}$ and concentrations of the drug typically ranged from $1 \mathrm{nM}$ to $0.1 \mathrm{mM}$ in 10-fold steps. After a $10 \mathrm{~min}$ pre-incubation of the enzyme and inhibitor, hydrolysis of the Ellman's reagents (ATChI and DTNB) was monitored for $10 \mathrm{mins}$ at $405 \mathrm{~nm}$, in a Dynex 96-well plate reader (Dynex, Chantilly, VA, USA). Percent residual AChE activity values (relative to the control) were plotted in Prism ${ }^{\circledR}$ (GraphPad, USA) and analyzed by non-linear regression (curve-fit) to generate $\mathrm{IC}_{50}$ values. Half maximal inhibitory concentration $\left(\mathrm{IC}_{50}\right)$ is defined as the inhibitor concentration that blocks $50 \%$ of the enzyme activity and is used to define the potency of the inhibitor. Mosquito selectivity (S) was determined by the $\mathrm{IC}_{50}$ ratio of $\mathrm{hAChE} / \mathrm{AgAChE}$ for each compound. Statistical significance of both potency and toxicity of the compounds was assessed based on non-overlapping of $95 \%$ confidence intervals ( $95 \% \mathrm{CI})$.

\subsection{Insect Bioassays}

A standard WHO-treated filter paper assay [13] was used to assess contact toxicity of the structural analogs, dissolved in ethanol and without any added silicon or other oil, which reduced activity (data not shown). An initial range finding assay was performed with concentrations of $0.1,0.5$, and $1 \mathrm{mg} / \mathrm{mL}$ 
of each compound, to identify additional test concentrations needed to obtain an $\mathrm{LC}_{50}$ value. From this initial assay, only compounds showing greater than $50 \%$ mortality at $0.5 \mathrm{mg} / \mathrm{mL}$ were tested further, with up to five concentrations per compound and 25 mosquitoes (2-5-day old sugar-fed females) per treatment. Assays were repeated at least twice using different batches of mosquitoes to account for inter-batch variability. Treated mosquitoes and untreated controls were maintained at $75 \% \mathrm{RH}$ and $25.6{ }^{\circ} \mathrm{C}$. Mortality was assessed after $24 \mathrm{~h}$ and corrected for control mortality by the method of Abbott [14]. Data was plotted in Poloplus ${ }^{\circledR}$ and a probit analysis performed to generate LC $_{50}$ values; the carbamate concentration that killed $50 \%$ of the exposed mosquitoes. Statistical significance of different $\mathrm{IC}_{50}$ values was judged by non-overlap of the $95 \%$ confidence intervals.

\section{Results}

\subsection{AChE Potency and Mosquito Toxicity of Terbam (1a) Analogs}

We have previously disclosed results with terbam (3-t-butylphenyl methylcarbamate) 1a. It displayed excellent potency for inhibiting $A g A C h E$ and excellent contact toxicity to $A n$. gambiae $\left(\mathrm{LC}_{50}=37 \mu \mathrm{g} / \mathrm{mL}\right)$, but enzymatic inhibition selectivity (S) of only 12-fold [5]. Except for 2a, all modifications using $\mathbf{1 a}$ as a template involved holding the 3-t-butyl group constant. Bromination at the 4-position (1b) led to a 16-fold loss in $A g A C h E$ inhibitory potency, compared to a 4-fold loss in hAChE inhibitory potency, relative to 1a (Table 1). Bromination at the 6-position (1d) increased inhibitory potency to both AChEs (about 2-fold for $A g$ AChE and 7-fold for hAChE), but reduced toxicity to mosquitoes 7-fold (Table 1). A 6-iodo substituent (1e) led to 58-fold and 2-fold loss in potency to $A g A C h E$ and hAChE, respectively, and a significant loss of mosquitocidal activity. Finally, addition of a 6-hexyl group (1f) drastically reduced $A g A C h E$ inhibitory potency while only reducing hAChE inhibitory potency 20-fold (Table 1).

Installation of a 4-carboxamido group abrogated $A g A C h E$ inhibition and mosquito toxicity (1h-11). For hAChE, however, we observed micromolar $\mathrm{IC}_{50}$ values with the allyl (1h), N-di-ethyl (1k), and $N$-ethyl (11) carbamoyl analogs, but no activity was observed with the methyl (1i) and iso-butyl (1j) analogs (Table 1). Addition of a thioether functionality at C6, as exemplified by compounds $\mathbf{1} \mathbf{m}-\mathbf{1 0}$, caused significant loss of $A g A C h E$ inhibition potency; 27 -fold with $\mathbf{1 m}$ and much more with $1 \mathrm{n}$ and 1o, and they had little insecticidal activity. Finally, when the tert-butyl group of 1a was replaced with the smaller and highly electron-withdrawing trifluoromethyl moiety (2a), inhibitory potency to both mosquito and human AChEs was lost, but surprisingly the compound was toxic to mosquitoes, although it was less toxic than 1a by about 10-fold (Table 1). 
Table 1. AChE inhibition and mosquito toxicity to adult female An. gambiae by 3-tert-butylphenyl carbamate 1a and the substituted analogs shown in Figure 1.

\begin{tabular}{|c|c|c|c|c|}
\hline Compound & $A g \mathrm{AChE}, \mathrm{IC} 50,{ }^{\mathrm{a}} \mathrm{nM}$ & hAChE ${ }^{\mathrm{a}} \mathrm{IC} 50, \mathrm{nM}$ & $S^{b}$ & LC $_{50}$ or $\%$ Mortality $^{c}$ \\
\hline $1 a^{d}$ & $36(34-38)^{a}$ & * $320(293-349)^{a}$ & 9 & $37(14-60)^{a}$ \\
\hline $1 b$ & $580(260-892)^{b}$ & * $1200(913-1533) b$ & 2 & $600(570-627)^{b}$ \\
\hline $1 d$ & $14(12-17)^{\mathrm{c}}$ & ${ }^{*} 48(40-56)^{c}$ & 3.4 & $260(239-276)^{c}$ \\
\hline $1 e$ & $2100(1195-2309)^{d}$ & * $640(538-752) d$ & 0.3 & $20 \%$ \\
\hline 1f & $>10^{5}$ & $6300(5126-7460)^{\mathrm{e}}$ & $<0.06$ & $4 \%$ \\
\hline $1 \mathrm{~h}$ & $>10^{5}$ & $2800(1719-4677)^{f, g}$ & $<0.03$ & $0 \%$ \\
\hline $\mathbf{1 i}$ & $>10^{5}$ & $>10^{5}$ & - & $4 \%$ \\
\hline $\mathbf{1 j}$ & $>10^{5}$ & $>10^{5}$ & - & $0 \%$ \\
\hline $1 \mathrm{k}$ & $>10^{5}$ & $2300(1189-4448)$ b,f.g & $<0.023$ & $0 \%$ \\
\hline 11 & $>10^{5}$ & $5500(2830-8128)^{e, f, g}$ & $<0.06$ & $0 \%$ \\
\hline $1 \mathrm{~m}$ & $970(830-1172)^{b}$ & * $640(572-719) d$ & 0.66 & $0 \%$ \\
\hline 1n & $32,000(23,060-63,300)^{e}$ & * $4700(3777-5784)$ e,f & 0.15 & $4 \%$ \\
\hline 10 & $75,000(\mathrm{~N} / \mathrm{A})$ & $2200(1653-2941)^{g}$ & 0.03 & $16 \%$ \\
\hline $2 a$ & $>10^{5}$ & $>10^{5}$ & - & $390(333-471)^{d}$ \\
\hline
\end{tabular}

${ }^{a} \mathrm{IC}_{50}$ with $(95 \% \mathrm{CI}){ }^{\mathrm{b}} \mathrm{S}=$ selectivity ratio $\left(\mathrm{IC}_{50} \mathrm{hAChE} / \mathrm{Ag} \mathrm{AChE}\right)$, and and asterisk indicates an $\mathrm{IC}_{50}$ value for $\mathrm{hAChE}$ that is significantly different from $\mathrm{AgAChE}$, as judged by non-overlap of the $95 \% \mathrm{CI}^{\mathrm{C}}{ }^{\mathrm{C}} \mathrm{LC}_{50}$ in $\mu \mathrm{g} / \mathrm{ml}$ or $\%$ mortality at $1 \mathrm{mg} / \mathrm{mL} ; \mathrm{IC}_{50}$ s or $\mathrm{LC}_{50} \mathrm{~s}$ within a column not labeled by the same lower case letter are significantly different $(p>0.05)$, as judged by non-overlap of the $95 \%$ CI.<smiles>CNC(=O)Oc1cccc(C(C)(C)C)c1</smiles>

1 a<smiles>C=CCNC(=O)c1ccc(OC(=O)NC)cc1C(C)(C)C</smiles>

$1 \mathrm{~h}$<smiles>CNC(=O)Oc1ccc(Br)c(C(C)(C)C)c1</smiles>

$1 b$<smiles>CNC(=O)Oc1cc(C(C)(C)C)ccc1Br</smiles>

1d<smiles>CNC(=O)Oc1cc(C(C)(C)C)ccc1I</smiles>

1e<smiles>CCCSc1ccc(C(C)(C)C)cc1OC(=O)NC</smiles>

$1 f$<smiles>CNC(=O)Oc1cc(C(C)(C)C)ccc1S(C)(=O)=O</smiles>

$1 \mathrm{~m}$<smiles>CNC(=O)Oc1ccc(C(=O)NC)c(C(C)(C)C)c1</smiles>

$1 \mathbf{i}$<smiles>CNC(=O)Oc1ccc(C(=O)NCC(C)I)c(C(C)(C)C)c1</smiles>

1j<smiles>CNC(=O)Oc1cc(C(C)(C)C)ccc1SP(F)I</smiles>

10<smiles>CCN(C)C(=O)c1ccc(OC(=O)NC)cc1C(C)(C)C</smiles>

11

Figure 1. Terbam (1a) and structural analogs described in this study.

\subsection{SAR of 3-Trialkylsilyl-and 2-Thioalkyl-Substituted Methylcarbamates}

Close structural analogs of $\mathbf{1 a}$ featured replacement of the $t$-butyl group with trialkylsilyl groups, and these analogs had similar inhibition potency and selectivity, but reduced contact toxicity as exemplified by $\mathbf{3} \mathbf{a}$ and $\mathbf{4 a}$, which were reported in a previous study [5]. Thus, analogs of $\mathbf{3 a}$ and $\mathbf{4 a}$, variants featuring 4-methyl (3c, $4 \mathbf{c})$ and 5-fluoro (3g, $\mathbf{4 g}$ ) were prepared (Figure 2) and assayed (Table 2). 
Both substitutions reduced $A g A C h E$ inhibition potency, and 5-fluorination had a significantly more deleterious effect for the 3-SiMe 3 derivative than for the 3-SiEtMe $\mathrm{S}_{2}$ derivative (3g and $\mathbf{4 g}$ ). None of these variants had improved enzymatic selectivity, and reductions in AgAChE inhibition potency correlated with reduced mosquitocidal action (Table 2).<smiles>CNC(=O)Oc1cccc([Si](C)(C)C)c1</smiles>

3a<smiles>CNC(=O)Oc1ccc(C)c([Si](C)(C)C)c1</smiles>

$3 c$<smiles>CNC(=O)Oc1cc(F)cc([Si](C)(C)C)c1</smiles>

3g<smiles>CC[Si](C)(C)c1cccc(OC(=O)NC)c1</smiles>

$4 a$<smiles>CC[Si](C)(C)c1cc(OC(=O)NC)ccc1C</smiles>

4c<smiles>CC[Si](C)(C)c1cc(F)cc(OC(=O)NC)c1</smiles>

$4 g$<smiles>CCC(CC)CSc1ccccc1OC(=O)NC</smiles>

$5 a$<smiles>CCCSc1ccccc1OC(=O)NC</smiles>

$6 a$

Figure 2. Analogs of 3-trialkylsilyl- and 2-thioalkyl-substituted methylcarbamates.

One additional 2-thioalkylphenyl methylcarbamate (6a) (Figure 2) was also synthesized based upon previous studies that identified compound 5a [5], as having good enzyme selectivity (135-fold), but poor contact toxicity on paper (Table 2). As can be seen, 2-thiopropylphenyl methylcarbamate $6 \mathbf{a}$ had a 3-fold reduction of inhibitory potency to $A g A C h E$, and 7-fold reduced inhibition selectivity, but had improved paper toxicity to adult An. gambiae (Table 2).

Table 2. Enzyme inhibition potency and toxicity to adult female An. gambiae of chemical structures shown in Figure 2.

\begin{tabular}{|c|c|c|c|c|}
\hline Compound & $A g \mathrm{AChE} \mathrm{IC}{ }_{50}{ }^{\mathrm{a}} \mathrm{nM}$ & $\mathrm{hAChE} \mathrm{IC}_{50}{ }^{\mathrm{a}}, \mathrm{nM}$ & $S^{b}$ & $\mathrm{LC}_{50}{ }^{\mathrm{c}}$ or $\%$ Mortality $^{\mathrm{c}}$ \\
\hline $3 \mathbf{a}$ & $50(40-64)^{a}$ & * $490(452-526)^{a}$ & 9.8 & $170(162-176)^{a}$ \\
\hline $3 c$ & $380(318-450)^{b}$ & * $2400(2032-2814)^{b}$ & 6.3 & $240(154-330)^{\mathrm{ac}}$ \\
\hline $3 g$ & $2600(1260-5453)^{\mathrm{c}}$ & $4200(3714-4616)^{c}$ & 1.6 & $4 \%$ \\
\hline $4 a$ & $72(67-78)^{d}$ & * $630(515-761)^{a, d}$ & 8.8 & $190(154-229)^{\mathrm{a}}$ \\
\hline $4 c$ & $630(556-714)^{\mathrm{e}}$ & * $1400(1205-1559) \mathrm{e}$ & 2.2 & $16 \%$ \\
\hline $4 g$ & $190(158-225)^{\mathrm{f}}$ & * $1900(1649-2245)^{\mathrm{f}}$ & 10 & $440(405-474)^{b}$ \\
\hline $5 a$ & $37(32-43)^{a}$ & * $5000(4514-5618) \mathrm{g}$ & 135 & $27 \%$ \\
\hline $6 \mathbf{a}$ & $110(135-152)^{g}$ & * $2300(2019-2596)^{\mathrm{f}}$ & 21 & $340(321-361)^{c}$ \\
\hline
\end{tabular}

${ }^{\mathrm{a}} \mathrm{IC}_{50}$ with $(95 \% \mathrm{CI}){ }^{\mathrm{b}} \mathrm{S}=$ selectivity ratio $\left(\mathrm{IC}_{50} \mathrm{hAChE} / \mathrm{AgAChE}\right)$, and and asterisk indicates an $\mathrm{IC}_{50}$ value for hAChE that is significantly different from $A g A C h E$, as judged by non-overlap of the $95 \% \mathrm{CI} ;{ }^{\mathrm{c}} \mathrm{LC} 50$ in $\mu \mathrm{g} / \mathrm{mL}$ or $\%$ mortality at $1 \mathrm{mg} / \mathrm{mL} ; \mathrm{IC}_{50} \mathrm{~s}$ or $\mathrm{LC}_{50} \mathrm{~s}$ within a column not labeled by the same lower case letter are significantly different $(p>0.05)$, as judged by non-overlap of the $95 \% \mathrm{CI}$.

\subsection{Toxicity of N-Sulfenylated Methylcarbamates 7a-e}

$N$-Sulfenylated methylcarbamates (Figure 3) were also tested because these pro-insecticides have been shown to have lower mammalian toxicity; hydrolysis of the labile sulfur-nitrogen bond in vivo yields the active insecticide [15]. N-Sulfenylated analogs of $\mathbf{1 a}, \mathbf{3 a}$, and $\mathbf{3 c}$ were synthesized using thiophenyl or para-substituted thiophenyl groups (7a-7e). Among the $N$-sulfenylated compounds, only $\mathbf{7 a}$ and $\mathbf{7 b}$ showed contact toxicity to mosquitoes in the WHO paper assay. It was observed that 7a killed $70 \%$ of G3 mosquitoes in $24 \mathrm{~h}$ at $0.5 \mathrm{mg} / \mathrm{mL}$ but was not toxic at $0.1 \mathrm{mg} / \mathrm{mL}$. Likewise, $7 \mathbf{b}$ had 100\% G3 mortality in $24 \mathrm{~h}$ at $1 \mathrm{mg} / \mathrm{mL}$ but was not toxic at $0.5 \mathrm{mg} / \mathrm{mL}$. Despite showing modest 
paper contact toxicity, $7 \mathbf{a}$ was highly toxic to G3 mosquitoes when topically applied, with an $\mathrm{LD}_{50}=$ $2.0 \mathrm{ng} /$ female and $95 \% \mathrm{CI}$ of $1.8-2.3$.<smiles>CN(C)C(=O)Oc1ccccc1S(=O)(=O)N(C)C</smiles>

$7 a$<smiles>Cc1ccc(SN(C)C(=O)Oc2cccc(C(C)(C)C)c2)cc1</smiles>

$7 b$

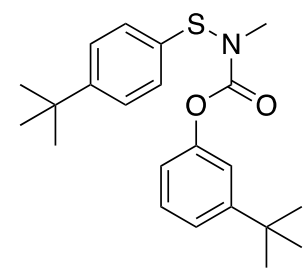

7c<smiles>CN(Sc1ccccc1)C(=O)Oc1cccc([Si](C)(C)C)c1</smiles>

7d<smiles>Cc1ccc(OC(=O)N(C)Sc2ccccc2)cc1[Si](C)(C)C</smiles>

$7 e$

Figure 3. $N$-sulfenylated pro-insecticidal analogs of 3-t-butyl-phenyl- and 3-trimethylsilyl-phenyl methylcarbamates.

\section{Discussion}

This study initiated a synthesis and testing program with the goal of expanding the existing SAR as it pertains to carbamate inhibition of $A g A C h E$ and hAChE, as well as toxicity. Due to the overall limited number of analogs made for each chemical series, this work constitutes a search for new leads as opposed to a thorough exploration of SAR of these two enzymes. It is noteworthy that much of the classical literature on carbamates focused on activity against housefly AChE and topical toxicity to this species, as well as larval mosquito bioassays, which makes comparisons to the data of the present study somewhat difficult.

\subsection{Effects of Structural Modification of 1a: Implications for the AgAChE Active Site}

The AChE-ligand interactions and molecular docking experiments with structurally diverse carbamates have been extensively explored in previous studies $[4,5,11]$. Examining the data in Table 1, it is clear that $A g \mathrm{AChE}$ can accommodate only relatively small substituents at the 6-position of terbam (1a). A 6-bromo-substituent (1d) is favorable, but the slightly larger 6-iodo (1e) and 6-thiomethyl (1m) substituents adversely impact $A g A C h E$ inhibitory potency. The larger 6-hexyl (1f), 6-isobutylthio (1n), and 6-isopropylthio (1o) groups all have significantly reduced potency for inhibition of AgAChE. Interestingly, hAChE inhibitory potency is less sensitive to steric bulk at the 6-position. In previous studies, SAR of 3-substituted phenyl carbamates suggested that $A g A C h E$ had a slightly larger ligand binding pocket than $\mathrm{hAChE}$ in order to accommodate a 3-t-butyl group [5]; the present study suggests that in the vicinity of the 6-position, hAChE is more accommodating to steric bulk than $A g A C h E$. The design of the 4 -carboxamido analogs $\mathbf{1 h}-\mathbf{l}$ was inspired by preliminary molecular modeling (data not shown) that suggested a hydrogen-bond acceptor at the $\mathrm{C} 4$ position of $1 \mathrm{a}$ might increase inhibition potency. Unfortunately, these compounds were even less potent inhibitors of $\mathrm{AgAChE}$ than the 4-bromo analog $\mathbf{1 b}$. The last analog of $\mathbf{1 a}$ explored was $\mathbf{2 a}$, which replaced the $3-t-\mathrm{Bu}$ with $3-\mathrm{CF}_{3}$. We propose that the drastic decrease in inhibition potency can be attributed to electronic effects. Sterically, the $\mathrm{CF}_{3}$ group was approximately intermediate in size compared to the isopropyl and $t$-butyl groups [16], so there should be plenty of space for it in the AChE active site. Thus, the poor inhibition potency of $\mathbf{2 a}$ may be due to repulsive interaction of the electron-rich $\mathrm{CF}_{3}$ group with one or more of the aromatic amino acid sidechains that are present in the active site of $A g A C h E$ [4]. Reviewing the hAChE potency of these inhibitors in Table 1, none of these modifications improved enzymatic selectivity, and in many cases reversed the selectivity to be more potent against hAChE, which was especially true of the carbamoylated phenyl ring analogs, $\mathbf{1 h}-\mathbf{1}$. Lastly, in general, the decreases seen in $A g A C h E$ inhibition potency were reflected by decreased An. gambiae contact toxicity. Interestingly, however, compound 2a exhibited only a 10-fold decrease in contact toxicity relative to 1a, despite its drastically reduced (>300-fold) $A g$ AChE inhibition potency. A convincing explanation for this discrepancy remains elusive at present. 
The AChE-ligand interactions and molecular docking experiments with structurally diverse carbamates have been extensively explored in previous studies $[4,5,11]$. Examining the data in Table 1 , it is clear that $A g \mathrm{AChE}$ can accommodate only relatively small substituents at the 6-position of terbam (1a). A 6-bromo-substituent (1d) is favorable, but the slightly larger 6-iodo (1e) and 6-thiomethyl (1m) substituents adversely impact $A g A C h E$ inhibitory potency. The larger 6-hexyl (1f), 6-isobutylthio (1n), and 6-isopropylthio (1o) groups all have significantly reduced potency for inhibition of AgAChE. Interestingly, hAChE inhibitory potency is less sensitive to steric bulk at the 6-position. In previous studies, SAR of 3-substituted phenyl carbamates suggested that $A g A C h E$ had a slightly larger ligand binding pocket than $\mathrm{hAChE}$ in order to accommodate a 3-t-butyl group [5]; the present study suggests that in the vicinity of the 6-position, hAChE is more accommodating to steric bulk than AgAChE. The design of the 4-carboxamido analogs $1 \mathrm{~h}-1$ was inspired by preliminary molecular modeling (data not shown) that suggested a hydrogen-bond acceptor at the $\mathrm{C} 4$ position of $\mathbf{1 a}$ might increase inhibition potency. Unfortunately, these compounds were even less potent inhibitors of $A g \mathrm{AChE}$ than the 4-bromo analog $\mathbf{1 b}$. The last analog of $\mathbf{1 a}$ explored was $\mathbf{2 a}$, which replaced the $3-t-\mathrm{Bu}$ with $3-\mathrm{CF}_{3}$. We propose that the drastic decrease in inhibition potency can be attributed to electronic effects. Sterically, the $\mathrm{CF}_{3}$ group was approximately intermediate in size compared to the isopropyl and $t$-butyl groups [16], so there should be plenty of space for it in the AChE active site. Thus, the poor inhibition potency of $2 a$ may be due to repulsive interaction of the electron-rich $\mathrm{CF}_{3}$ group with one or more of the aromatic amino acid sidechains that are present in the active site of $A g A C h E$ [4]. Reviewing the hAChE potency of these inhibitors in Table 1, none of these modifications improved enzymatic selectivity, and in many cases reversed the selectivity to be more potent against $\mathrm{hAChE}$, which was especially true of the carbamoylated phenyl ring analogs, $\mathbf{1 h}-\mathbf{1}$. Lastly, in general, the decreases seen in $A g A C h E$ inhibition potency were reflected by decreased An. gambiae contact toxicity. Interestingly, however, compound 2a exhibited only a 10-fold decrease in contact toxicity relative to $\mathbf{1 a}$, despite its drastically reduced (>300-fold) $\mathrm{AgAChE}$ inhibition potency. A convincing explanation for this discrepancy remains elusive at present.

\subsection{Structural Modification of Trialkylsilylphenyl Methylcarbamate and 2-Thioalkyl Methylcarbamates}

Turning to analogs of the $\mathbf{3 a}$ and $\mathbf{4 a}$, variants featuring 4-methyl (3c, $4 \mathbf{c})$ and 5-fluoro $(3 \mathbf{g}, \mathbf{4 g})$ were prepared and assayed. Both substitutions reduced $A g A C h E$ inhibition potency, notably, 5-fluorination had a significantly more deleterious effect for the 3-SiMe 3 derivative than for the 3-SiEtMe ${ }_{2}$ derivative ( $3 \mathrm{~g}$ and $4 \mathrm{~g}$ ). None of these variants had improved enzymatic selectivity, and reductions in $A g \mathrm{AChE}$ inhibition potency correlated with reduced mosquitocidal action.

A single variant of 2-thioalkyl-substituted 5a featured a smaller alkyl group (propyl, 6a). This compound proved less potent and selective for inhibition of $A g \mathrm{AChE}$, relative to 5a. Interestingly, the insecticidal potency of $\mathbf{6} \mathbf{a}$ improved relative to $\mathbf{5} \mathbf{a}$, which may reflect reduced oxidative metabolism in the mosquito or better transfer off of paper. In a previous study of ortho thioalkyl phenylcarbamates, compound 6a had essentially optimal activity in this series for both housefly AChE inhibition and Culex pipiens larval toxicity [3]. Moreover, the addition of piperonyl butoxide to housefly toxicity bioassays increased toxicity, indicating that sulfur oxidation was not bioactivating as it is for aldicarb [3]; on the contrary, these results suggest oxidative detoxication.

\subsection{Paper versus Topical Toxicity of N-Sulfenylated N-Methylcarbamates 7a-e}

$\mathrm{N}$-arylsulfenyl and $\mathrm{N}$-alkylsulfenyl derivatives of methylcarbamate insecticides have been shown to possess lower mammalian toxicity and be more effective mosquito larvicides than the parent methylcarbamates [15]. This same study also reported that the selective toxicity did not relate directly to anticholinesterase activity. Thus, selectivity of these compounds was likely due to differential metabolism. Toxicity of arylsulfenylated compounds to mice was lowered up to 50-fold in these compounds, but was 5- to 17-fold lower for alkylsulfenylated analogs [15]. With such confirmed improvement of mammalian safety, as well as increased toxicity to insects, a new generation of 
sulfenylated compounds was synthesized for testing. However, sulfenylated analogs of $\mathbf{1 a}, \mathbf{3 a}$, and $\mathbf{3 c}$ did not show good paper contact toxicity to mosquitoes, but 7a was twice as toxic to G3 mosquitoes topically, compared to 1a, its non-sulfenylated parent. The topical toxicity of the other compounds has not been determined, and the lack of paper contact toxicity of $\mathbf{7 a}$, even though it is highly toxic when topically applied, cannot be fully addressed at this point

\section{Conclusions}

Structural modifications of promising anticholinesterase mosquitocides $\mathbf{1 a}, \mathbf{3 a}, \mathbf{4 a}$, and $\mathbf{5 a}$ were examined in this paper. A series of 6-substituted analogs of 1a analogs indicated AgAChE cannot accommodate a substituent larger than bromine in this position. A group of 4-substituted analogs of 1a, 3a, and 4 indicates that $A g A C h E$ cannot accommodate any of the attempted substitutions in this position. Replacement of the t-butyl group of $1 \mathrm{a}$ with $\mathrm{CF}_{3}$ resulted in poor anticholinesterase activity, but surprisingly this compound (2a) did have measurable mosquitocidal activity. The 2-thiopropyl analog of 5a (6a) possessed lower $A g A C h E$ inhibition potency but improved toxicity to adult mosquitoes. Finally, $N$-sulfenylated proinsecticidal derivatives of $\mathbf{1 a}$ and $\mathbf{3 a}$ that were synthesized had poor contact activity on paper, but one of them (7a) had good topical activity.

Author Contributions: Conceptualization, S.D., M.T., P.R.C., and J.R.B.; methodology, J.M.M.; software, M.T.; validation, J.M.M.; formal analysis, J.M.M.; investigation, J.M.M.; resources, M.M., Q.-H.C., J.A.H., and D.M.W.; data curation, J.M.M.; writing—original draft preparation, J.M.M.; writing—review and editing, P.R.C. and J.R.B.; visualization, J.M.M., M.T., P.R.C., and J.R.B.; supervision, M.T., P.R.C., and J.R.B.; project administration, M.T., P.R.C., and J.R.B.; funding acquisition, M.T., P.R.C., and J.R.B.

Funding: This research was funded by the FNIH, grant number 1496, under the Grand Challenges in Global Health program.

Acknowledgments: The authors are grateful to the MR4 as part of the BEI Resources Repository, NIAID, NIH, for providing eggs for the Anopheles gambiae G3 (MRA-112) strain.

Conflicts of Interest: The authors declare no conflict of interest. The funders had no role in the design of the study; in the collection, analyses, or interpretation of data; in the writing of the manuscript, or in the decision to publish the results.

\section{References}

1. Ware, G.W.; Whitacre, D.M. The Pesticide Book, 6th ed.; MeisterPro Information Resources: Willoughby, OH, USA, 2004; pp. 59-60. ISBN 1892829-11-8.

2. WHO. WHO Recommended Insecticides for Indoor Residual Spraying against Malaria Vectors. World Health Organization. Available online: https://www.who.int/neglected_diseases/vector_ecology/vectorcontrol/Insecticides_IRS_2_March_2015.pdf (accessed on 26 April 2019).

3. Metcalf, R.L. Structure-activity relationships of carbamates. Bull. World Health Organ. 1971, 44, 43-78. [PubMed]

4. Carlier, P.R.; Anderson, T.D.; Wong, D.M.; Hsu, D.C.; Hartsel, J.; Ma, M.; Wong, E.A.; Choudhury, R.; Lam, P.C.; Totrov, M.M.; Bloomquist, J.R. Towards a species-selective acetylcholinesterase inhibitor to control the mosquito vector of malaria, Anopheles gambiae. Chem.-Biol. Interact. 2008, 175, 368-375. [CrossRef] [PubMed]

5. Hartsel, J.A.; Wong, D.M.; Mutunga, J.M.; Ma, M.; Anderson, T.D.; Wysinski, A.; Islam, R.; Wong, E.A.; Paulson, S.L.; Li, J.; et al. Re-engineering aryl methylcarbamates to confer high selectivity for inhibition of Anopheles gambiae versus human acetylcholinesterase. Bioorg. Med. Chem. Lett. 2012, 22, 4593-4598. [CrossRef] [PubMed]

6. Wong, D.M.; Li, J.; Chen, Q.H.; Han, Q.; Mutunga, J.M.; Wysinski, A.; Anderson, T.D.; Ding, H.; Carpenetti, T.L.; Verma, A.; et al. Select small core structure carbamates exhibit high contact toxicity to "carbamate-resistant" strain malaria mosquitoes, Anopheles gambiae (Akron). PLoS ONE 2012, 7, e46712. [CrossRef] [PubMed]

7. Wong, D.M.; Li, J.; Lam, P.C.; Hartsel, J.A.; Mutunga, J.M.; Totrov, M.; Bloomquist, J.R.; Carlier, P.R. Aryl methylcarbamates: Potency and selectivity towards wild-type and carbamate-insensitive (G119S) Anopheles 
gambiae acetylcholinesterase, and toxicity to G3 strain An. gambiae. Chem.-Biol. Interact. 2013, 203, 314-318. [CrossRef] [PubMed]

8. Kolbezen, M.J.; Metcalf, R.L.; Fukuto, T.R. Insecticide structure and activity, insecticidal activity of carbamate cholinesterase inhibitors. J. Agric. Food Chem. 1954, 2, 864-870. [CrossRef]

9. Fahmy, M.A.H.; Metcalf, R.L.; Fukuto, T.R.; Hennessy, D.J. Effects of deuteration, fluorination and other structural modifications on the carbamoyl moiety upon the anticholinesterase and insecticidal activities of N-methylcarbamates. J. Agric. Food Chem. 1966, 14, 79-83. [CrossRef]

10. Park, B.K.; Kitteringham, N.R.; O'Neill, P.M. Metabolism of fluorine-containing drugs. Annu. Rev. Pharmacol. Toxicol. 1966, 41, 443-470. [CrossRef] [PubMed]

11. Anderson, T.D.; Paulson, S.L.; Wong, D.M.; Carlier, P.R.; Bloomquist, J.R. Pharmacological mapping of the acetylcholinesterase catalytic gorge in mosquitoes with bis(n)-tacrines. In ACS Symposium Series; Oxford University Press: Oxford, UK, 2009; Volume 1014, pp. 143-151. [CrossRef]

12. Ellman, G.L.; Courtney, K.D.; Andres, V.J.; Feather-Stone, R.M. A new and rapid colorimetric determination of acetylcholinesterase activity. Biochem. Pharmacol. 1961, 7, 88-95. [CrossRef]

13. WHO. Guidelines for Determining the Susceptibility or Resistance of Insects to Insecticides; World Health Organization: Geneva, Switzerland, 1981; ISBN 978-92-4-150515-4.

14. Abbot, W.S. A method of computing the effectiveness of an insecticide. J. Econ. Entomol. 1925, 18, $265-267$. [CrossRef]

15. Black, A.L.; Chiu, Y.-C.; Fahmy, M.A.H.; Fukuto, T.R. Selective toxicity of N-sulfenylated derivatives of insecticidal methylcarbamate esters. J. Agric. Food Chem. 1973, 21, 747-751. [CrossRef] [PubMed]

16. Meanwell, N.A. Synopsis of some recent tactical application of bioisosteres in drug design. J. Med. Chem. 2011, 54, 2529-2591. [CrossRef] [PubMed]

(C) 2019 by the authors. Licensee MDPI, Basel, Switzerland. This article is an open access article distributed under the terms and conditions of the Creative Commons Attribution (CC BY) license (http://creativecommons.org/licenses/by/4.0/). 